\title{
Role of platelet-rich fibrin in enhancing the viability of autologous microlobular fat graft: an animal-based experimental study
}

Trimartani Koento, Mirta Hediyati Reksodiputro, Olvi Nancy Marimpan

pISSN: 0853-1773 • elSSN: 2252-8083 https://doi.org/10.13181/mji.v28i3.2538 Med J Indones. 2019;28:246-51

Received: March 12, 2018

Accepted: August 28, 2019

Authors' affiliations: Department of Otorhinolaryngology, Faculty of Medicine, Universitas Indonesia, Cipto Mangunkusumo Hospital, Jakarta, Indonesia

\section{Corresponding author:}

Trimartani Koento

Department of Otorhinolaryngology,

Faculty of Medicine, Universitas

Indonesia, Cipto Mangunkusumo

Hospital, Jalan Diponogoro No.71,

Kenari, Senen, Central Jakarta 10310, DKI

Jakarta, Indonesia

Tel/Fax: +62-21-3910701

E-mail: trimartani@yahoo.com.au

\begin{abstract}
BACKGROUND Tissue fat has been used as a fat graft in plastic reconstructive surgery since ancient times by surgeons. However, an issue with this usage is that fat gets absorbed by up to $30-50 \%$, especially centrifuged fats. The aim of this study was to compare the survival of microlobular fat, centrifuged fat, microlobular fat with plateletrich fibrin (PRF), and centrifuged fat with PRF.
\end{abstract}

METHODS Subcutaneous fat was taken from the stomach area and divided into two parts, microlobular fat and centrifuged fat, and processed by adding PRF as follows: 1 ) microlobular fat without PRF; 2) microlobular fat with PRF; 3) centrifuged fat without PRF; and 4) centrifuged with PRF. These fat grafts were implanted into rabbit earlobes. Each procedure was performed repeatedly nine times for a total of 36 repetitions for all groups.

RESULTS Microscopic evaluation revealed the highest number of adipocytes in the microlobular fat with PRF group (mean = 1,932) and the highest number of fibroblasts (mean $=600.5$ ) compared to those in other treatment groups, although the difference was not statistically significant. There was more neovascularization in the microlobular fat group (mean $=228.5$ ), but it was not statistically significant.

CONCLUSIONS Addition of PRF to microlobular fat graft enhances the number of adipocytes and fibroblasts, including the enhancement of neovascularization.

KEYWORDS fatty tissue, platelet-rich fibrin, survival of fat
Primary closure of defect will create tissue contracture around the adjacent area of defect. ${ }^{1}$ Silicone, either in liquid or solid form, is frequently used by surgeons. However, silicone presents several complications such as scar tissue, unwanted pigmentation, and skin thinning, and it may also predispose to cancer., ${ }^{1,2}$ After the development of plastic surgery, the popularity of fat graft is relatively new, although the concept of fat transfer is not new in the field of plastic reconstructive surgery. In early 1893 , free fat was used by Neuber ${ }^{2}$ to fill in the soft tissue defect. In 1909, the use of autologous abdominal fat was reported to correct the defect in the malar and cheek area. The application of the fat graft technique was then increasing with the increasing popularity of liposuction in the 1980s. This was due to the natural properties of fat and several positive reports of fat grafting being published. ${ }^{2-4}$ Fat has a unique consistency, softness, and nature and host compatibility, and it does not cause any additional cost to patients. It can be used to correct soft tissue defects and any other cosmetic contour deformities. ${ }^{3}$ However, till date, the primary issue of this technique is the less predictable long-term outcome. Unfortunately, over time, the transplant gets absorbed by up to $30-50 \%$, encouraging the 
need for autologous materials to support the viability of the graft. Excess fat use with the addition of platelet-rich fibrin (PRF) to prevent absorption which is could be to solve the problem in plastic reconstruction surgery.

PRF is an autologous material that possesses several growth factors such as platelet-derived growth factor- $A B$, transforming growth factor $\beta_{1}$, and vascular growth factor that can help maintain fat viability. 4,5 These factors can stimulate cell and matrix proliferation as well as angiogenesis. In fat tissue, PRF stimulates angiogenesis, which is necessary to maintain viability. ${ }^{6-9}$ Angiogenesis, immunity, and epithelial cover are the three important phenomena for healing and soft tissue maturation. PRF can simultaneously support the development of these three phenomena. A study reported by Neuber was the first to describe excellent aesthetic results of filling a retracted scar at the infraorbital rim. This study was conducted to evaluate the use of PRF in enhancing the viability of microlobular fat graft.

\section{METHODS}

This study was approved by the Ethical Committee of the Faculty of Medicine, Universitas Indonesia (No. 83/UN2.F1/ETIK/2017). The rabbit animal model was used in this study.

\section{Study duration and location}

This study was performed at the Department of Anatomical Pathology, Faculty of Medicine, Universitas
Indonesia, Cipto Mangunkusumo Hospital, for a duration of 4 weeks.

\section{Study design}

An animal-based experimental research design was applied in this study using 6 rabbits, each in four different treatment groups. The New Zealand White rabbit species weighing $2.5-3.0 \mathrm{~kg}$ were used in this study. The autologous fat grafts were implanted into each rabbit's ear, which was further divided into eight groups (Figure 1), resulting in a total of 48 groups. Each group was assigned to one of the following treatments: microlobular fat, centrifuged fat, microlobular fat with PRF, and centrifuged fat with PRF. The rabbits were followed up for a period of 4 weeks. Grafted tissues were resected and evaluated microscopically for the number of adipocytes and fibroblasts and neovascularization using the ImageJ program developed by the National Institutes of Health.

\section{Fat graft preparation}

Subcutaneous fat was taken from the stomach area, separated into two parts, sliced into microlobular shape, and centrifuged at 1,200g. PRF was obtained from the peripheral blood collected from rabbit ears and then centrifuged at 1,500g. The microlobular fat was subdivided into two parts, comprising $0.5 \mathrm{ml}$ that was directly implanted into the rabbit's ear and another $0.5 \mathrm{ml}$ that was prefabricated with PRF and then implanted. The centrifuged fat was also divided into two parts, comprising $0.5 \mathrm{ml}$ that was directly
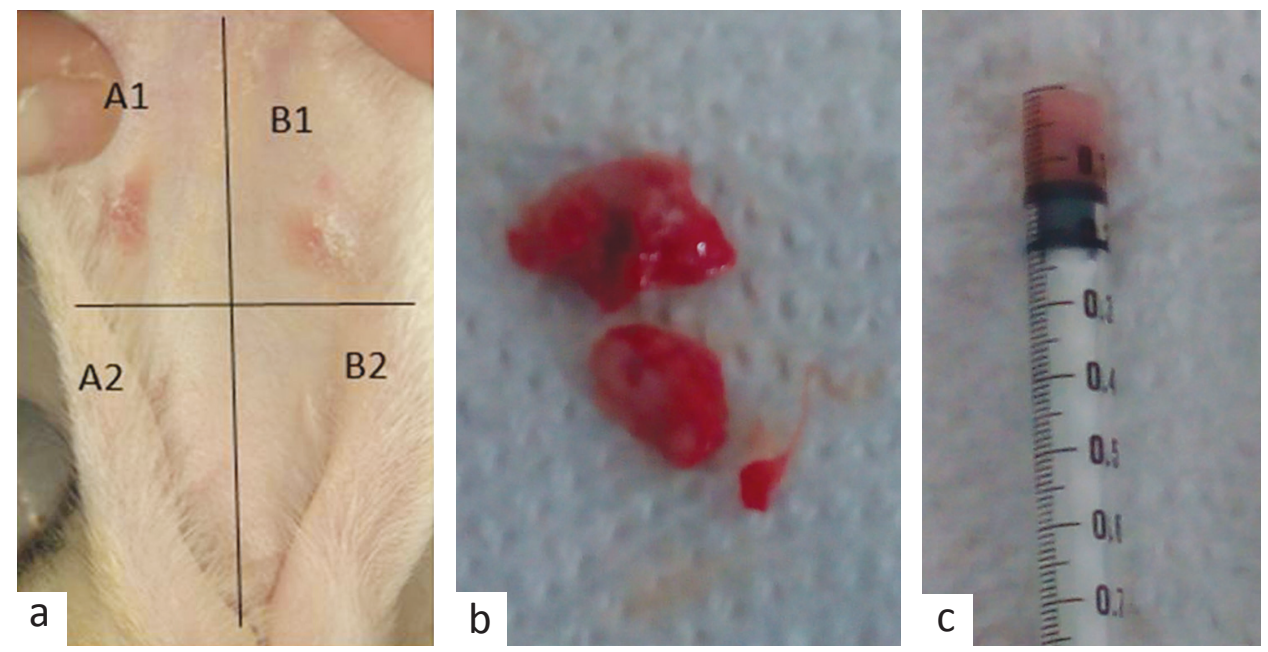

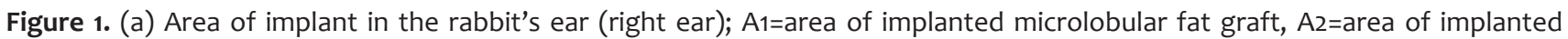
microlobular fat graft with PRF, B1=area of implanted centrifuged fat, B2=area of implanted centrifuged with PRF; (b) Microlobular fat with PRF; (c) Centrifuged fat with PRF. PRF=platelet-rich fibrin 
implanted and another $0.5 \mathrm{ml}$ that was mixed with PRF and then implanted.

\section{Evaluation}

Microscopic parameters were only measured in the fourth week (Figure 2). Fatty tissue was extracted and stained using hematoxylin and eosin. The ImageJ software was used for the evaluation of adipocytes, fibroblasts, and neovascularization by measuring the width of the area. After staining, the specimen slides are viewed under a microscope with a magnification of $40 x$, then taken five photo per slices. Peripheral counts were done per field of view, and the number of fibroblasts and neovascularization were marked by the endothelium around the adipocytes. In addition, the presence or absence of an inflammatory reaction characterized by monocytes, lymphocytes, and macrophages on the specimen slides was evaluated, but the investigator did not count the total number of cells.

\section{Statistical analysis}

All results were compared between the treatment groups to determine significant differences among them. Post-hoc analysis of variance was used to analyze the results of the microscopic evaluation between the treatment groups.

\section{RESULTS}

There were six experimental rabbits, but one died after intervention due to infection. The implanted fat was blunt-dissected and measured. The fat diameter was measured using a caliper, and the number of adipocytes and fibroblasts and neovascularization were evaluated microscopically and analyzed using the ImageJ software.

There was no statistically significant difference between the four groups in the number of adipocytes and fibroblasts and neovascularization ( $p>0.05)$. However, with the ImageJ software analysis, the total number of adipocytes in the microlobular fat with PRF groups (mean =1,932) was found to be higher than that in the microlobular fat without PRF, centrifuged fat, or centrifuged fat with PRF groups (Figure 2a). Furthermore, the number of fibroblasts in the microlobular fat with PRF groups (mean $=600.5$ ) was higher than that in the other groups (Figure 2b). However, neovascularization was more in the a

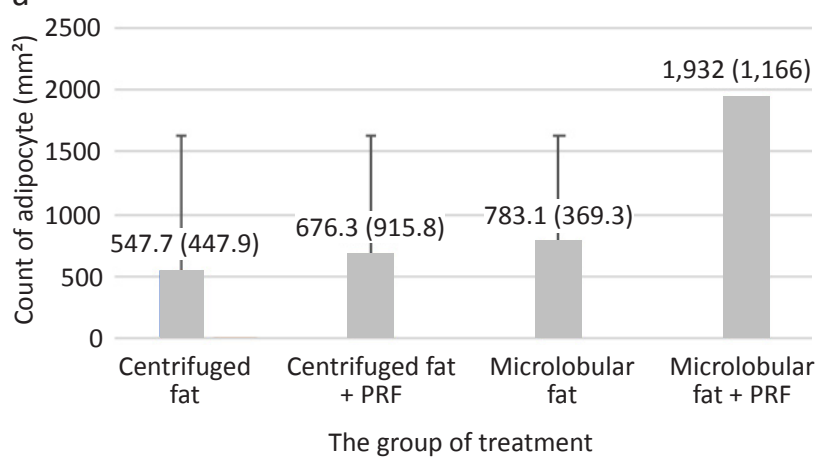

b

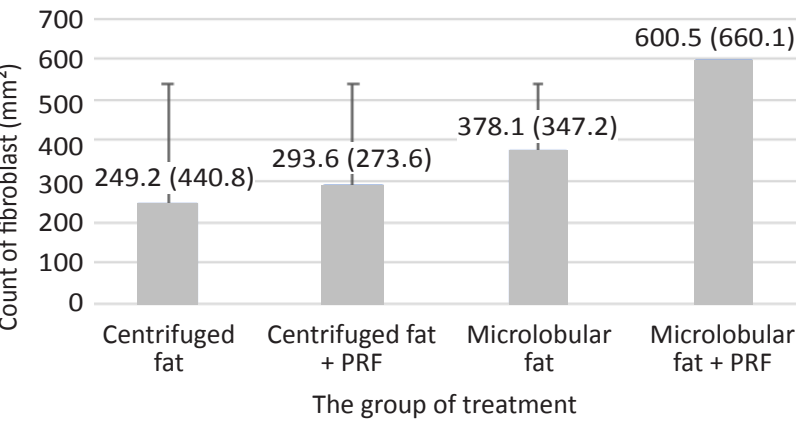

C

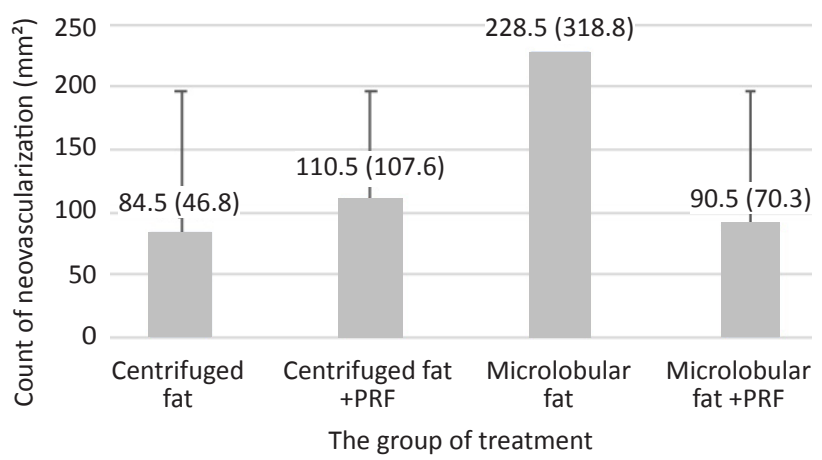

Figure 2. Overview of the number in each group analyzed using ImageJ: (a) adipocytes; (b) fibroblasts; and (c) neovascularization. PRF=platelet-rich fibrin

microlobular fat without PRF groups (mean $=228.5)$ than that in the other groups (Figure 2c). Post-hoc analysis was conducted to assess the possibility of a statistically significant difference among the four groups, but no significant difference was detected among the four groups ( $p>0.05)$, either in the number of adipocytes $(p=0.5)$ and fibroblasts $(p=0.3)$ or in neovascularization $(p=0.2)$. This lack of difference could be due to the small sample size. However, with the ImageJ software analysis, a significantly greater number of adipocytes and fibroblasts was obtained in the microlobular fat treatment group with the addition of PRF (Figure 2).

Figure 3 shows the histological results of the fat grafts in all groups. A greater adipocyte count was 

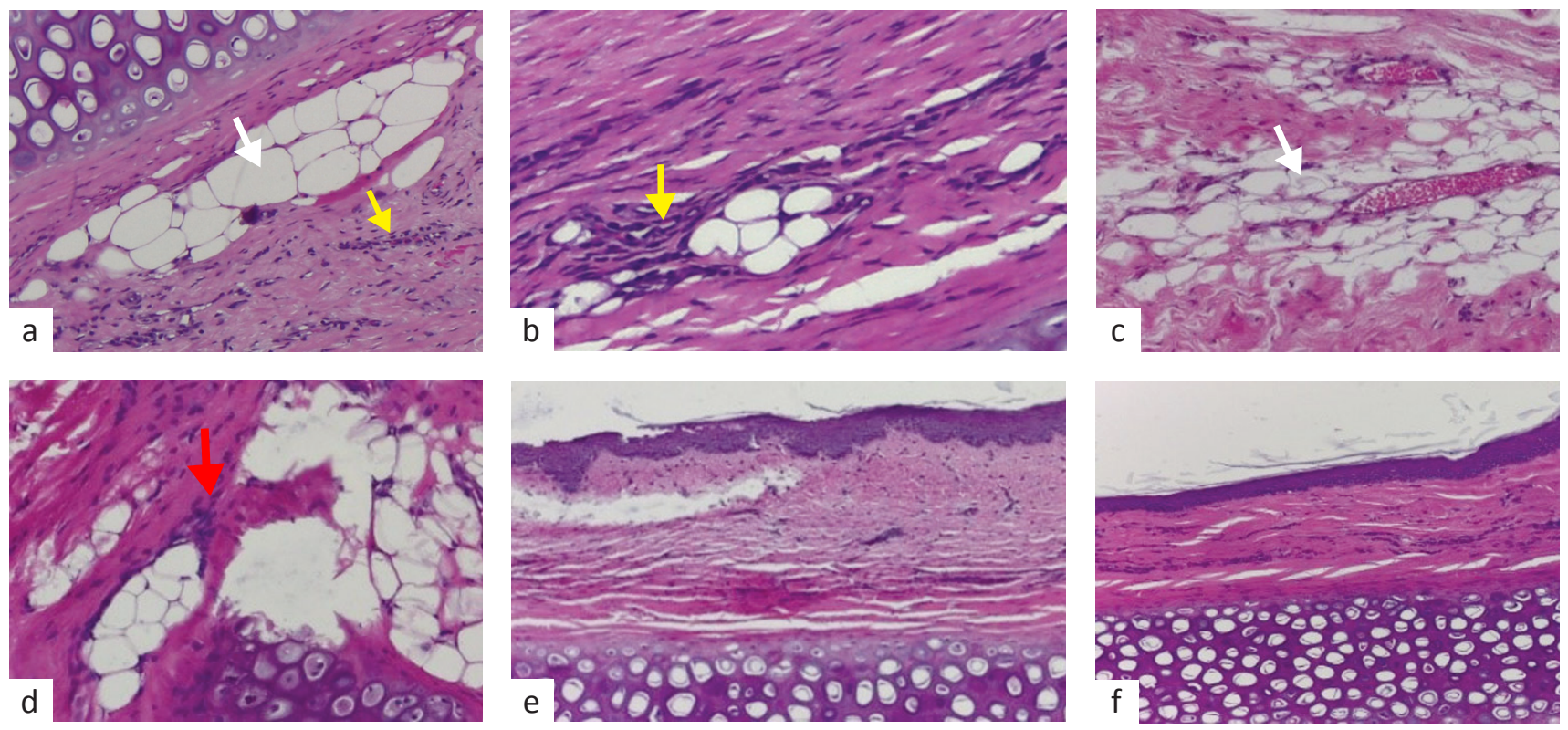

Figure 3. Histological features of (a) microlobular fat without PRF show collection of fat (white arrow) with only a few inflammatory cells (yellow arrow), (b) centrifuged fat surrounded by numerous fibroblasts and minimal endothelium (yellow arrow), (c) microlobular fat + PRF with increased number of fat cells (white arrow), (d) centrifuged fat with PRF with increased number of fibroblasts (red arrow), (e) PRF, (f) rabbit's ear dermired

observed in the microlobular fat group with PRF than that in other groups. Figure za shows microlobular fat without PRF, which depicts a collection of fat (white arrow), only slightly surrounded by fibroblasts, with only a few inflammatory cells (yellow arrow). Figure $3 \mathrm{~b}$ shows centrifuged fat without the addition of PRF, which depicts a slightly fatty appearance and surrounded by numerous fibroblasts and minimal endothelium (yellow arrow). Figure $3 c$ shows microlobular fat with the addition of PRF, wherein fat clusters are surrounded by more endothelium showing new neovascularization and some fibroblasts (white arrow). Figure $3 \mathrm{~d}$ depicts centrifuged fat with the addition of PRF, which shows fat surrounded by some fibroblast (red arrow).

\section{DISCUSSION}

The primary problem that arises after the implantation of a fat graft is the absorption of adipose tissue, considering that after 3 weeks of fat grafting, the rate of adipose tissue absorption is $30-70 \%$. Fat is often used as an autologous material because it does not cause allergic reactions, is easy to obtain, and the amount is quite large. However, over time, fat gets easily absorbed; hence, an autologous material that also possesses various growth factors is needed to maintain the viability of fat. This high rate of absorption may require additional grafts, which implies the need for repeated harvest procedures and an increase in cost and surgical risks as well pain and discomfort for the patient. The retention of fat grafting is known to be affected by its size, and numerous applications of autogenous platelet-rich plasma have been investigated for enhancing the survival of free fat grafts; however, only a few studies have investigated the application of PRF.9-14

In this study, the microlobular fat with PRF group had the largest number of adipocytes (mean $=1,932$ ) compared to that in the centrifuged fat, microlobular fat, and centrifuged fat with PRF groups, although the difference wa s not statistically significant. Condé-Green et $a^{9}$ reported a study using two methods of fat extraction by scraping (microlobular fat) and lipoaspiration that were later centrifuged. He isolated mesenchymal stem cells (MSCs) from the adipose fat tissue and found that microlobular fat had more intact adipose stem cells (ASCs) than centrifuged lipoaspirated fat, because the integrity of adipocytes in the microlobular fat is better maintained. ${ }^{8}$ Although centrifugation create concentrated adipose tissue and removed red blood cells, but the centrifugation make a destroyed most of the adipocytes. Centrifugation significantly destroys most of the adipocytes, and adipose-derived stem cells reduce graft uptake at the recipient area. 
Raposio et al ${ }^{12}$ reported that centrifuged fat contains concentrated fat, which is separated from blood, lipids, proteases, and other components, that can reduce the quality of the implanted fat. Centrifuged fat reduces the amount of fat volume, thus requiring a greater implant volume. ${ }^{9-11}$

Lu et al ${ }^{14}$ confirmed that ASCs present in the grafted fat contain vascular endothelial growth factor (VEGF), that show by the amount of capillary. This implies that vascularization determines the viability of fat and VEGF plays an important role in neovascularization of the recipient's area. Clinically, the best selection in fat implants is to use microlobular fat, on the basis of the fact that ASCs in microlobular fats are intact compared to those in non-centrifuged fat. ${ }^{13-16}$

Some PRF-related studies have shown that proliferation of pre-adipocytes occurs immediately when mixed with PRF. In addition, PRF would speed up the proliferation of endothelial cells. Estimation analysis (regression) revealed that the increase in neovascularization was directly proportional to the increase in fat volume, which was observed to be $72 \%$, indicating that neovascularization can predict $72 \%$ of viability of the fat graft. Addition of PRF to centrifuged fat and optimization of centrifugal force may increase the quality of the implanted fat and will also probably lead to better long-term results. Current studies have shown that adipose tissue is an abundant source of MSCs, which improve the outcome in wound healing and maintain the adipocyte quantity.3,9,17-23

The clinical impact of this study in plastic reconstructive surgery is that it provides surgeons with knowledge about the use of fats as autologous substances for reconstruction and replacement of silicone substances that can cause various negative reactions in the body. This study is expected to contribute knowledge about autologous materials to maintain the viability of fat implants. It is also expected that reconstructive plastic surgeons could obtain information about PRF for maintaining the survival of the implant.

This study evaluated the role of PRF in enhancing the viability of autologous microlobular fat graft using an animal experimental model. Although the results were positive, there were statistical limitations, with the sample size being small and the evaluation being conducted for only 4 weeks. According ethical issue, the used of experimental animals is restricted.

\section{Conclusions}

This study reported no significant finding, although the microscopic evaluation after 4 weeks revealed that the microlobular fat group with the addition of PRF had more adipocyte counts and more neovascularization than the microlobular fat without PRF groups. This study shows the use PRF in fat tissue as an implant can improve the survival of viability implant.

\section{Conflict of Interest \\ The authors affirm no conflict of interest in this study.}

\section{Acknowledgment}

The completion of this research was achieved by the cooperation by several people; thanks to Alida Harahap, MD, Clinical Pathologist, $\mathrm{Ph}$. D., who helped in calculating the required $\mathrm{CaCl}_{2}$ molarity and for all corrections in this paper. The author is grateful to Ms Riesye, Dwi, and Saptawati and to Mr. Doli, Slamet for their assistance and advice during this research.

\section{Funding Sources \\ This study was funded by the DRPM PITTA grant, Universitas Indonesia.}

\section{REFERENCES}

1. Rizaliyana S, Perdanakusuma DS. An experience using fat graft and wire scalpel in reconstructing lower limb defect [thesis]. Surabaya (Indonesia); 2012. Indonesian.

2. Pu LL. Towards more rationalized approach to autologous fat grafting. J Plast Reconstr Aesthet Surg. 2012;65(4):413-9.

3. Xie Y, Zheng DN, Li QF, Gu B, Liu K, Shen GX, et al. An integrated fat grafting technique for cosmetic facial contouring. J Plast Reconstr Aesthet Surg. 2010;63(2):270-6.

4. Keyhan SO, Hemmat S, Badri AA, Abdeshahzadeh A, Khiabani K. Use of platelet-rich fibrin and platelet-rich plasma in combination with fat graft: which is more effective during facial lipostructure? J Oral Maxillofac Surg. 2013;71(3):610-21.

5. Maione L, Vinci V, Klinger M, Klinger FM, Caviggioli F. Autologous fat graft by needle: analysis of complications after 1000 patients. Ann Plast Surg. 2015;74(3):277-80.

6. Ren Y, Kolonin MG, Li Y. Fat grafting with adipose stem cells: the successes and challenges. Cell Stem Cells Regen Med. 2015;1(2).

7. Cohen SR, Strem BM. Comparison of three different fat graft filtration in a closed system. PRS J. 2013;873-80.

8. Modarressi A. Platelet rich plasma (PRP) improves fat grafting outcomes. World J Plast Surg. 2013;2(1):6-13.

9. Condé-Green A, Baptista LS, de Amorin NF, de Oliveira ED, da Silva KR, Pedrosa Cda S, et al. Effects of centrifugation on cell composition and viability of aspirated adipose tissue processed for transplantation. Aesthet Surg J. 2010;30(2):49-55.

10. Conti G, Jurga M, Benati D, Bernardi P, Mosconi E, Rigotti G, et al. Cryopreserved subcutaneous adipose tissue for fat graft. Aesth Plast Surg. 2015;39(5):800-17.

11. Kunachak S, Chayangsu P. Concept of Adipose Tissue Engineering and Adult Human Stem Cells. In: Handbook of Masterclass Rhinoplasty. 2012. pp. 55-8.

12. Raposio E, Simonacci F, Perrotta RE. Adipose-derived stem cells: comparison between two methods of isolation for clinical applications. Ann Med Surg. 2017;20:87-91.

13. Ågren MS, Rasmussen K, Pakkenberg B, JørgensenB. Growth factor and proteinase profile of Vivostat ${ }^{\circledR}$ platelet-rich fibrin linked to issue repair. Vox Sang. 2014;107(1):37-43.

14. Lu F, Li J, Gao J, Ogawa R, Ou C, Yang B, et al. Improvement of the survival of human autologous fat transplantation by using 
VEGF-transfected adipose-derived stem cells. Plast Reconstr Surg. 2009;124(5):1437-46.

15. Kølle SF, Fischer-Nielsen A, Mathiasen AB, Elberg JJ, Oliveri RS, Glovinski PV, et al. Enrichment of autologous fat grafts with ex-vivo expanded adipose tissue-derived stem cells for graft survival: a randomized placebo-controlled. Lancet. 2013;382(9898):1113-20.

16. Sclafani AP, McCormick SA. Induction of dermal collagenesis, angiogenesis, and adipogenesis in human skin by injection of platelet-rich fibrin matrix. Arch Facial Plast Surg. 2012;14(2):1326.

17. Reksodiputro M, Widodo D, Bashiruddin J, Siregar, Malik S. PRMF enhance wound healing process. Facial Plast Surg. 2014;30:670-5.

18. Singh A, Kohli $M$, Gupta N. Platelet rich fibrin: a novel approach for osseous regeneration. J Maxillofac Oral Surg. 2012;11(4):4304.

19. Ehrenfest DMD, Bielecki T, Mishra A, Borzini P, Inchingolo F,
Sammartino G, et al. In search of a consensus terminology in the field of platelet concentrates for surgical use: platelet-rich plasma (PRP), platelet-rich fibrin (PRF), fibrin gel polymerization and leukocytes. Curr Pharm Biotechnol. 2012;13(7):1131-7.

20. Goldfarb RM, Shapiro AL. Benefits of autologous fat grafting using fat mixed with platelet rich fibrin matrix (PRFM) selphyl. Am J Cosmet Surg. 2012;29(1):62-4.

21. Sugihara $H$, Funatsumaru S, Yonemitsu N, Miyabara S, Toda S, Hikichi Y. A simple culture method of fat cells from mature fat tissue fragments. J Lipid Res. 1989;30(12):1987-95.

22. Eto H, Kato H, Suga H, Aoi N, Doi K, Kuno S, et al. The fate of adipocytes after nonvascularized fat grafting: evidence of early death and replacement of adipocytes. Plast Reconstr Surg. 2012;129(5):1081-92.

23. Ko MS, Jung JY, Shin IS, Choi EW, Kim JH, Kang SK, et al. Effects of expanded human adipose tissue-derived mesenchymal stem cells on the viability of cryopreserved fat grafts in the nude mouse. Int J Med Sci. 2011;8(3):231-8. 